\title{
Clinician Perspectives on Group Visits for Advance Care Planning Among Caregivers and Older Adult Patients With Heart Failure
}

\author{
Alexis Coulourides Kogan, PhD, MSG, Kya Kraus, MPH, Bonnie Olsen, PhD, \\ Julia I. Bandini, PhD, and Sangeeta C. Abluwalia, PhD
}

Background: Advance care planning (ACP) is critical for older adults with heart failure; however, patient-level and clinician-level barriers exist. Although a group visit (GV) approach to engage patients in ACP has proven effective among general geriatric populations, little is known about clinician perceptions/likelihood of referral.

Methods: Qualitative study to understand clinician perspectives on GVs for ACP among older adult patients with heart failure and caregivers. Twenty physicians and advance practice providers participated in telephone-based interviews guided by a semistructured research protocol. Transcripts were analyzed using a grounded theory approach.

Results: Results highlight variability in clinician engagement in ACP but greater agreement around the factors that prompt discussions. Qualitative themes included (1) inherent properties of GVs (characteristics that make GVs ideal for most but less ideal for some, risk-to-benefit ratio); (2) purpose of GVs (general education, "priming the pump" for subsequent discussions, providing tools for action); and (3) format and procedures for GVs (inclusion/exclusion considerations, organizing by unifying characteristic, link back to clinicians).

Conclusions: This is the first study to gain clinician insights into ACP GVs specific to patients and caregivers affected by heart failure. Results shed light on an important topic and suggest key considerations for conducting GVs for ACP. (J Am Board Fam Med 2021;34:375-386.)

Keywords: Advance Care Planning, Cardiology, Caregivers, Family Medicine, Grounded Theory, Group Medical Visits, Health Communication, Heart Failure, Internal Medicine, Interview, Los Angeles, Palliative Medicine, Primary Care, Qualitative Research

\section{Introduction}

Advance care planning (ACP) is critical for older adults with heart failure, who face an uncertain yet unmistakably progressive trajectory, marked by frequent exacerbations and complex treatment choices. ${ }^{1}$

This article was externally peer reviewed.

Submitted 1 June 2020; revised 19 October 2020; accepted 20 October 2020.

From the Keck School of Medicine of USC, University of Southern California, Dept. of Family Medicine and Geriatrics, Alhambra (ACK, KK, BO); RAND Corporation, Boston, MA (JIB); RAND Corporation, Santa Monica, CA (SCA).

Funding: This study was funded by the National Institute on Aging of the National Institutes of Health under grant R21-AG053716.

Conflict of interest: The study team report having no conflicts of interest.

Corresponding author: Alexis Coulourides Kogan $\mathrm{PhD}$, MSG, University of Southern California Keck School of Medicine, Alhambra, CA 91803 (E-mail: acoulour@usc. edu).
This trajectory is markedly different from that of patients with cancer. Planning for future care needs and discussing one's values and goals for treatment with health care providers, early and often over the course of the illness, can help to assure person-centered and preference-concordant care. ${ }^{2}$

Despite efforts to improve ACP, several barriers persist, including clinician level (lack of time during clinic visits, uncertainty around the optimal time to raise ACP, and inadequate ACP communication training), and patient/family level (reluctance to contemplate end of life, difficulty identifying health care values and goals without adequate context, and limited health literacy around complex treatment choices and trade-offs). ${ }^{3,4}$ Patients with heart failure in particular may perceive ACP as unimportant because of misconceptions around the life- 
limiting nature of their disease. ${ }^{5,6}$ In addition, patients experience multiple care transitions and see various providers over the course of a progressive illness, making it difficult to have regular ACP discussions that foster a shared understanding of a patient's goals. ${ }^{7}$

Group visits, where several patients with heart failure can meet together with a single clinician to share their disease experiences and discuss ACP, while accessing a range of values and preferences against which to consider and clarify their own health care goals, can establish a foundation from which more treatment-specific one-on-one patientprovider conversations can begin. Group visits can also offer important social support to empower patients to action and increase self-efficacy, while reinforcing information receiving during clinic visits. ${ }^{8,9}$ Heart failure is an exemplar condition for other life-limiting, variable-trajectory illnesses in that it can serve as an important blueprint for understanding how group visits for ACP could be used in this context. Some preliminary work ${ }^{10,11}$ points to the promise of group visits in facilitating more efficient and effective ACP within our resource-constrained health system environment. Yet, little is known about clinician perceptions of group visits for ACP and the likelihood of referral.

As part of a larger study to develop and test a group visit intervention for ACP among older adults with heart failure, ${ }^{12}$ we sought to understand clinician perceptions of group visits for ACP. Specifically, we were interested in learning how clinicians caring for older adults with heart failure currently engaged in ACP, including any challenges they face that could effectively be addressed through group visits, as well as their perceptions of how best to structure group visits, what ACP content might be most appropriate for a group visit, and for which patients might a group visit be beneficial.

\section{Methods}

To achieve these aims, we conducted a prospective qualitative study among clinicians to elicit their input on group visits for ACP among older adult patients with heart failure. The Institutional Review Boards of the RAND Corporation and the University of Southern California each approved this study. The study report follows the Con- solidated Criteria for Reporting Qualitative Studies Guidelines. ${ }^{13}$

\section{Procedures and Sampling}

Convenience sampling among physicians (MDs and DOs) and advance practice providers (NPs and PAs) from multiple departments who typically (or who would be expected to) have patients with heart failure in their panels were approached for this study. This included the departments of Family Medicine, Internal Medicine, Cardiology, Pulmonology/Critical Care, and Palliative Medicine at the clinical site conducting this study. In addition, convenience sampling among internal medicine clinicians of patients who participated in the group visits was employed. Subsequent snowball sampling by way of referral from other study participants was used to identify and invite potential cardiology and internal medicine participants. From September to November 2019, 30 clinicians (10 from family medicine, 9 internal medicine, 9 cardiology, 1 pulmonology/critical care medicine, and 1 palliative care) were invited via e-mail to participate in an interview. For those who did not respond, a single reminder e-mail was sent 1 week following the initial invitation.

This qualitative study consisted of individual interviews conducted telephonically (by KK) or inperson (by ACK) with clinicians who responded to the study invitation. Both interviewers were members of the USC Department of Family Medicine where one third of clinician participants were also affiliated but did not have any other relationships with participants. ACK had prior research experience with ACP and recent experience with group visits from observing those visits as part of the larger study (in press). Interviews were guided by a 10-item semistructured research protocol developed by study investigators (SCA, BO, JIB, and $\mathrm{ACK}$ ) and pretested for content and face validity by 3 researchers (SCA, JIB, and ACK) and a geropsychologist (BO) with extensive clinical and research experience in ACP. The interviews aimed to elicit clinician perspectives on ACP among older adult patients with heart failure and the group visit format. Interview questions included clinician engagement with ACP including frequency of ACP discussions, topics addressed, and challenges faced, perceived utility of group visits for ACP, acceptable and unacceptable topics for the group visits, how group visits for ACP could support the clinician in subsequent interactions with his or her patient, and 
identification of appropriate patients to participate in a group visit for ACP. At the outset of each interview, clinicians were given background information on our larger study testing group visits for ACP for patients with heart failure and were read a description of a group visit for ACP that was included in the interview guide (see Appendix 1). Respondents were asked to consider their patients with heart failure while responding to the interview questions as opposed to their patients with other advanced illness. Verbal consent was obtained at the beginning of each interview. Interviews were audio recorded and transcribed verbatim. Concurrent coding allowed for study personnel to check for fidelity to the research model and protocol, rigor, and saturation. Data saturation was achieved after 14 interviews; however, 6 additional interviews were conducted for thoroughness and greater representation across the targeted medical specialties.

\section{Analysis}

Descriptive statistics were used to describe the sample using SPSS version 22. For the qualitative analysis, transcripts from each of the 20 interviews were analyzed using a grounded theory approach ${ }^{14,15}$ to identify and code themes that naturally emerged from the data (see Appendix 2). Two researchers (ACK and KK) independently coded the transcripts by noting ideas and relationships between concepts and categories and each identified a list of initial themes. Next, the 2 researchers convened to compare, discuss, sort, and refine the coding scheme, collapsing codes with similar meanings into distinct codes and subcodes. The authors jointly reviewed the revised codes and then each independently recoded the transcripts using these new codes. During this second independent coding, they also identified key phrases and passages that expressed distinct themes-ideas, descriptions, narratives, attitudes, beliefs, and values-and generated preliminary code lists. ${ }^{16}$ Finally, the researchers came together again to hold a consensus meeting to reconcile remaining coding differences to achieve greater than $90 \%$ consensus.

\section{Results}

Out of the 30 clinicians invited to participate in the study, 20 (67\%) agreed and completed an interview. Participants were most commonly a family medicine clinician $(\mathrm{n}=9 ; 5 \mathrm{MDs}, 1 \mathrm{DO}$, and 3 PAs)
Table 1. Clinician Characteristics

\begin{tabular}{lccc}
\hline & $\begin{array}{c}\text { Physician* } \\
(\mathrm{n}=13)\end{array}$ & $\begin{array}{c}\text { Advance Practice } \\
\text { Provider }^{\dagger} \\
(\mathrm{n}=7)\end{array}$ & $\begin{array}{c}\text { Overall } \\
(\mathrm{n}=20)\end{array}$ \\
\hline Family medicine & 6 & 3 & 9 \\
Internal medicine & 4 & 0 & 4 \\
Cardiology & 2 & 4 & 6 \\
Palliative care & 1 & 0 & 1 \\
\hline
\end{tabular}

${ }^{*} \mathrm{MD}, \mathrm{DO}$.

${ }^{\dagger} \mathrm{PA}, \mathrm{NP}$.

followed by cardiology ( $\mathrm{n}=6 ; 2$ MDs and 4 NPs), internal medicine ( $\mathrm{n}=4 ; 4 \mathrm{MDs})$, and palliative care $(\mathrm{n}=1 ; 1$ MD). See Table 1 . Ten clinicians did not respond to the invitation and were not included in the study. Out of the 30 clinicians, 12 had had a patient participate in an ACP group visit, although none were either aware that their patient participated or were aware of the outcome of the group visit. Overall, interviews lasted between 11 and 50 minutes $($ mean $=20.6 \pm 9.3$; median $=17.9)$.

\section{Clinician Engagement with ACP}

Clinician reports for engaging older adult patients with heart failure in ACP ranged from "rarely" to "every visit." The majority $(\mathrm{n}=14)$ of respondents indicated that they "should be better" about having these discussions and identified the annual wellness visit as the best time to bring up ACP, although some felt this was challenging due to the large physical assessment aspect of these visits. Family medicine clinicians also reported an initial patient visit as a good time to have an ACP discussion.

Clinicians reported discussing a wide variety of topics around ACP with patients with heart failure that ranged from goals of care and patients' values, to code status and choices for resuscitation and other life-sustaining measures. This variation was reported among clinicians from all specialties included in the study. Respondents who indicated having "many," or a "majority" of, older adult patients on their census reported having more values-based discussions focusing on goals of care, identifying a surrogate decision maker, and the advance directive form. Other respondents who reported caring for fewer older adult patients stated using the physician orders for life-sustaining treatment (POLST) form to guide their discussion and focusing on patient preferences around code status and other life-sustaining 
measures. In addition to the POLST-informed approach to ACP discussions, other reported strategies included bringing up broader planning topics such as home safety, the financial implications of declining functioning and independence, and personal assets and desires for estate distribution.

There was consistent agreement across all respondents and specialties around what would prompt the clinician to engage in an ACP discussion with a patient with heart failure. The most common reasons included change in health status/ decreased functioning, hospitalization ("recent"/ since the last visit), an upcoming surgery or major procedure, a new (serious) diagnosis, and if the patient initiated the conversation. Other reasons for engaging in ACP discussions with patients with heart failure were either more formalized situations (ie, annual wellness examination, requirement for upcoming move to an assisted living center, etc) or related to severity of illness/functional capacity (ie, declining memory, increasing care needs, advanced age, suffering/poor quality of life, patient and/or family frustration with physical decline and symptom burden, and lack of treatment options).

Clinicians reported numerous challenges with discussing ACP with their older adult patients with heart failure. The most common barriers were time constraints, patient lack of readiness (including resistance, avoidance, and deflecting the responsibility onto adult children), and patient-family issues or disagreement. One challenge reported to be shared by both clinicians and patients alike was lack of knowledge around ACP, especially when it came to the different forms (advance directive, POLST, and living will). While not all clinicians reported difficulty, some spoke specifically about experiencing challenges around discussing ACP with "healthy" or "well-functioning" patients overall, including those at advanced ages. Other reported challenges focused on patient-level issues such as low literacy and health literacy, reduced comprehension due to illness severity, cultural factors, language barriers, and lack of support for some patients (patients without family/friends or surrogate). Several cardiologists also spoke to challenges associated with vast variability in how their patients with heart failure perceive or accept their mortality with a diagnosis of heart failure. For example, they stated that their patients do not view a heart failure diagnosis as serious or life threatening as a cancer diagnosis.

\section{Clinician Perceptions of Group Visits for ACP}

Three broad, independent themes with subthemes relating to clinician perceptions of group visits for ACP for patients with heart failure were identified: (1) inherent properties of a group visit (characteristics that make a group visit an ideal format for most are also what makes them less ideal for some, and riskto-benefit ratio for group visits); (2) purpose of group visits (general education, "priming the pump" for subsequent discussions, providing the tools for action); and (3) format and procedures for group visits (participant inclusion and exclusion considerations, organizing groups by a unifying characteristic, and the link back to clinicians). See Table 2 .

\section{Inherent Properties of Group Visits}

The first theme focused on the inherent properties and nuances of a group visit for ACP. Respondents spoke to the nature and dynamic of a group visit as having inherent characteristics that hold important implications for discussing ACP. The "group" dynamic was credited for potentially providing support and comradery to attendees as well as creating a learning environment (both from the facilitator and other attendees), possible opportunity to open patients' minds to the topics and opinions of others, and allowing for patient-centered discussion. Many reported that a group visit for ACP could also be helpful for "priming the pump" for subsequent ACP discussions, increasing patient readiness to engage in ACP discussions, including family members or caregivers (valuable for both educating caregivers and helping family members get on the same page), and normalizing or destigmatizing the topic of ACP. Some clinicians felt that just offering a group to patients for ACP sends a positive message of support, implies value to (early) conversations, and is analogous to offering any other therapy or treatment to patients (it is part of the regular menu of services and would therefore never be denied as an option for someone).

For the same reasons that the group dynamic was viewed favorably, it was reported that it could also have drawbacks for some patients; specifically, patients who are introverted, private, and not comfortable being in a group setting or sharing among strangers. Clinicians were not in agreement when it came to excluding patients based on their personality and clinician-perceived comfort in a group setting, or encouraging all to attend but without expectations for open sharing or comment during 
Table 2. Clinician Perceptions of Group Visits for Advance Care Planning

\begin{tabular}{|c|c|c|}
\hline Theme & Participant ID & Quotation \\
\hline \multicolumn{3}{|c|}{ 1. Inherent properties of a group visit } \\
\hline & \multicolumn{2}{|c|}{ Double-edged sword } \\
\hline & 104 & $\begin{array}{l}\text { "I think the strength is that people can empathize with one another and once } \\
\text { they hear other people maybe vocalizing the same concerns or opinions, the } \\
\text { feel more comfortable in their own [situation] that they're not alone." }\end{array}$ \\
\hline & 106 & $\begin{array}{l}\text { "So I think that the education that can be done in a group visit would be very } \\
\text { helpful. But the discussion aspect of it, I think is going to depend on their } \\
\text { [participant's] personality and really how sort of extroverted versus } \\
\text { introverted they are: how comfortable they are." }\end{array}$ \\
\hline & 112 & $\begin{array}{l}\text { "I could see some unintended consequences of just upsetting the other } \\
\text { members [group participants] if things get too personal details in a group } \\
\text { setting. I mean, I think you want people to open up, but it's a balance. } \\
\text { There's a risk. Things could get too personal and too graphic." }\end{array}$ \\
\hline \multicolumn{3}{|c|}{ Risk-benefit ratio } \\
\hline & 102 & $\begin{array}{l}\text { "I think even people that feel like they wouldn't benefit from it, I feel like it's } \\
\text { something that they can benefit from." }\end{array}$ \\
\hline & 104 & $\begin{array}{l}\text { "I think it's a great idea [group visits for advance care planning]. I think it's } \\
\text { overdue and it'd really be nice if this can be accomplished outside of the } \\
\text { physician visit, at least the groundwork. . . Sometimes it's just really hard, } \\
\text { almost like pulling teeth, because people don't have a good understanding of } \\
\text { or are repressed thinking about their lack of independence, or their dying } \\
\text { days. And they're not forced to think about it because we, in American } \\
\text { society, want to always think about having a happy ending and think happy } \\
\text { thoughts." }\end{array}$ \\
\hline
\end{tabular}

Specialty

2. Purpose of group visits for advance care planning

Normalize

102

109

119

General education

104

105

108

Prime the pump

107
"I think one important thing is the setup; the way it's introduced and why it's being introduced and kind of painting a picture that this is something that is, should, be done with everybody, basically."

"When you make it [ACP] in a group setting, it sort of normalizes it and they [participants] can see examples of how its's used and why it's important and maybe [it] kind of reduces the stigma a little bit."

"People in the room [group visit participants] may have very different things going on with their health. I think just generally explaining the importance of advance care planning and the role that it plays in medical care is important, and I think as a way of kind of destigmatizing it that this is just a routine set of questions we ask everyone; I think that's fine. But beyond that I think making it work for everyone can be tricky."

"So I think the group discussion would be like... an introduction and opens up, 'What are you facing down the road?' And, 'what are the options in terms of once you lose your independence for living?' And, 'what are the options in terms of when you're looking at the end of your days?"”

"I think that a group visit would be helpful to disseminate general information more efficiently...I also see it being helpful, maybe having multiple people there for multiple questions and answers."

"Your goal [group visits] is just to expose them [participants to ACP], provide an avenue, and just hope they had a pleasant experience and maybe consider themselves more knowledgeable. But really not more, you know, like 'have decided one way or the other' necessarily.”

\footnotetext{
"A lot of patients are able to open up a little bit more, particularly when they are among their peers addressing their similar concerns and struggles. They're able to relate. . There's actually some therapeutic counseling element, as well, in that. And so I think in the context of advanced directive, if you're giving general information and kind of like discussing how individuals feel about it, that definitely is a primer to when they would maybe even discuss it with their physician or when they go to complete paperwork and discuss it with their family."
} 
Table 2. Continued

\begin{tabular}{|c|c|c|}
\hline Theme & Participant ID & Quotation \\
\hline & 112 & $\begin{array}{l}\text { "So maybe they [group visit participant] come to me with an advance directive } \\
\text { form [from the group visit] and have had some time to read it, digest it, and } \\
\text { then they come to me with specific questions about their situation. That } \\
\text { could be helpful. I think having them be aware of some of these trade-offs: } \\
\text { quality of life versus longevity-again, so that they're primed to speak about } \\
\text { these issues, goals of care issues_-could be helpful." }\end{array}$ \\
\hline
\end{tabular}

Specialty

Tools for action

113

"That group discussion [visit] could be just like where the patient could be a

Cardiol. springboard to introduce topics and let them research more topics. Gives you insight as to what they'd like to talk about and what issues they want addressed when they come to see you [clinician]."

"I think explaining to them what advance care planning is, what forms are recommended to have, how logistically do they accomplish getting those forms to be legal documents, would be really great before [the clinician] talking to them about it because then you could just focus on more of the substance of it. About what they would want rather than how do I make this happen once I've made my decision."

3. Format and procedures for group visits for advance care planning Inclusion/exclusion

"I think we're targeting older adults [for advance care planning] for good reasons, but really, it should be part of basic primary care. And an important part of it, I think."

"Anybody who I felt did not lack capacity to make decisions for him- or herself either due to mild cognitive impairment or diagnosed with dementia, I would not refer. . But other than that, I literally think that everybody could benefit from such a discussion."

[The most appropriate patients for ACP group visits] "I guess it would be the stable patients that we have already exhausted all of our treatment options and we're kind of managing them and just providing surveillance."

Organizing groups by a characteristic

"So you want to pick and choose your patients. Obviously you want to introduce the topic to everybody, but you want to really refer the people who are the most enthusiastic and could really augment a session and really ask in-depth questions and really move the discussion along."

"The patients who don't have a high risk of mortality in the next few years I don't think are appropriate for that kind of group therapy or group session."

"Well, it would be nice having people in similar circumstances brought together."

Link back to clinician

"I think just being sure that the paperwork's done and in the chart is basically all that really needs to happen."

"I think a general list of topics that were covered and then specifically after it [group visit] happens, a brief-and by "brief" I mean four or five bullet points-sort of the outcomes of the group visit. Did the group get sidetracked and not cover what a POLST form is? Did they not get past the quality of life versus longevity discussion? Did the conversation deteriorate? So four or five bullet points summarizing the extent to which topics were actually covered [documented] through the electronic health record.”

"I always believe that if you are able to educate patients about their disease process, give them the support that they need, provide them with resources, and connect the provider along with the patient and the family that they do the best."

"I would want to know what's going to be discussed in the visit and by who $[\mathrm{m}] . "$

ACP, Advance care planning; POLST, physician orders for life-sustaining treatment; Internal Medicine; FM, Family Medicine; Cardiol, Cardiology. 
the visit. Overall, participants expressed the potential for greater benefit versus harm for group visits for ACP.

\section{Purpose of Group Visits}

The second theme focused on the purpose clinicians felt an ACP group visit would serve for attendees. Overall, participants agreed that the group visit would serve 3 distinct purposes: (1) to "prime the pump" for subsequent ACP discussions and associated decision-making; (2) to provide basic education and increase knowledge around all aspects of ACP and related forms/formal documentation of wishes/ decisions; and (3) to promote action and follow-up by both attendees and clinicians. For example, attendees would be equipped with information and tools to make decisions and formally document their wishes, and clinicians could see a summary note of the visit in the electronic health record (EHR) so that they may continue the conversation and answer specific medical questions.

While many different topics for the group visits were suggested, the group consensus was to provide an overview of ACP and related topics. It was also specified that the facilitator should provide this information in an unbiased way and would simply provide facts and a general overview of topics. In addition, participants all agreed that it would be important to include a discussion (and possibly a list/ handout) of resources available to patients (ranging from senior centers, meals on wheels, palliative care, hospice, and long-term care). Other topic recommendations covered the full spectrum of end-oflife care and serious illness chronology, including forms (advance directive and POLST), finances, legal support, quality versus quantity of life, caregiver burden, naming a proxy, end-of-life care preferences and values, and tips on how to talk to your doctor. Topics that were identified as not appropriate for a group visit setting centered on specific medical details about individual group participants, including their diagnosis, prognosis/timeline, medications, treatments, or giving specific advice.

\section{Format and Procedures}

Finally, clinicians provided suggestions for the format and procedures for a group visit on ACP. Clinicians unanimously agreed that participants attending the group visits should be divided and organized by some unifying characteristic, although there was disagreement on exactly what the defining factor should be. Suggestions included clinician-perceived patient readiness, disease status/ severity (early stage, late stage, etc.), diagnosis, age, level of functioning, "healthy" versus "not healthy," language, culture, literacy level, and a group for patients and caregivers affected by Alzheimer disease or related dementias.

Clinicians reported feeling that many different patients constituted prime targets for ACP group visits but largely agreed that cognitively intact older adults, "sick" patients (ie, "most ill," "limited life expectancy"), and patients who had been recently hospitalized were the best candidates. Other suggestions ranged from a very specific type of patient to all patients. Recommendations included patients who are less connected or supported (eg, those with no family), diagnosed with cancer, ready and willing to attend and discuss ACP, unsure about but not against discussing $\mathrm{ACP}$, and those without completed documents (advance directive and POLST), who have chronic pain, and who are experiencing declining independence, and all patients.

To promote clinician follow-up to the group visit and patients' ACP actions, respondents largely suggested that the optimal link would be a summary note in the EHR. Other recommendations included a summary note sent via fax to their office or a physical document either mailed to their office, given to patients to bring to their next appointment, or both. Communication with the clinician's office beyond the EHR note was viewed as valuable because it could also inform the nurse or other aides to better ensure that the information got to the doctor or advance practice provider. Some respondents felt that specific information in addition to the group visit summary would be beneficial. For example, some suggested post-group visit "next steps," summary of patient wishes, proxy name and phone number, completed advance directive, code status, patient's reaction to the topic/visit, "red flags" that arose during the group visit, and patient's "action status" related to ACP. Finally, the creation of a single workflow for clinicians around ACP processes (ie, single place designated in EHR for scanned documents), referral to group visits, receiving reports after patients attend a visit, and a recommended approach for having ACP conversations (initial and follow-up) was recommended to standardize clinicians' approach and thoroughness. 


\section{Discussion}

This qualitative study aimed to understand the perspectives of clinicians on group visits for ACP among older adult patients with heart failure and their caregivers. To our knowledge, this is the first study to gain clinician insights into ACP group visits specific to patients and caregivers affected by heart failure. Results highlight the perceived utility of the group format for $\mathrm{ACP}$, the purpose of group visits for $\mathrm{ACP}$, and ideal procedures for conducting the group visits. Though clinicians were given the context of our work as being focused on older adult patients with heart failure and were asked to think about group visits for their patients with heart failure, it was unclear if some clinicians were responding more broadly about their patients with serious illness in general. However, responses still hold value as heart failure serves as an exemplar condition with a variable trajectory that holds implications for other noncancer conditions.

Clinician participants in our study perceived high utility for group visits for ACP among older adult patients with heart failure. These findings support previous research that studied the impact of group visits for ACP among general older adult primary care patients, ${ }^{10,11}$ patients from safety net settings aged 55 years or more, ${ }^{17}$ and patients with cancer ${ }^{18}$ where the groups visits were found to be effective. Mirroring the sentiments of our study participants, 2 studies also highlighted the value of the group nature of these visits. ${ }^{11,18}$ However, these studies also reported low attendance ${ }^{18}$ and a $40 \%$ participation rate, ${ }^{11}$, which may suggest that group visits are not acceptable for all patients, a sentiment also reported among clinician participants in our study. This finding warrants further investigation because, as suggested by several of the clinicians in our study, there may be value to introducing the topic of ACP and providing education to facilitate subsequent discussions and formal documentation down the line outside of a set study period.

In our study, clinicians overwhelmingly felt that the purpose for a group visit for ACP among older adult patients with heart failure and their caregiver would be to provide 3 elements: (1) a general introduction to the topic; (2) unbiased education around all aspects of important decisions; and (3) presentation of formal documents for subsequent completion (ie, advance directive and POLST). By offering group visits for $\mathrm{ACP}$ and focusing on these topics, it may convey an importance for ACP and normalize these conversations, which has been found to be associated with greater rates of advance directive completion. ${ }^{19}$ In addition, group visits for ACP among patients with heart failure may be key to bringing up a vital conversation that might otherwise not be taking place, especially due to the widespread phenomenon of patients with heart failure not viewing their condition as terminal..$^{20,21}$ For example, a recent study on the attitudes of oncologists, cardiologists, and primary care physicians toward ACP found that only $15 \%$ of cardiologists felt it was their responsibility to engage patients in ACP, compared with $68 \%$ of primary care physicians. $^{22}$ By using group visits to provide the education piece about ACP and the nuances of important decisions and documentation associated with it, clinicians may then be able to follow up with focused conversations that are more conducive to brief clinical encounters or dedicated, billable visits.

Previous research has shown that clinicians are more likely to use tangible, concrete items such as advance directive or POLST forms to engage patients in ACP. ${ }^{23}$ Therefore, a summary notice entered in the EHR, as suggested by the clinicians in our study, may be a way to not only inform clinicians about their patient's participation in an ACP group visit but may also serve as a point of discussion and action during subsequent medical visits. Additional research on ACP in general, and on group visits specifically, points to the importance of more than 1 meeting/conversation ${ }^{10,11,17,19,23,24}$ revisited over an extended period of time. ${ }^{19}$ This also suggests the need to standardize clinical workflows around $\mathrm{ACP}^{25}$ and incorporate information technology ${ }^{26}$ (ie, EHR triggers, reminders, summary notes, single location to upload completed ACP forms), a sentiment reported by our participants.

\section{Limitations}

While this study elicited important clinical insights into conducting group visits for ACP among older adult patients with heart failure, it is not without limitations. First, this study may be limited by including participants from 1 organization and only those that responded to our invitations. In addition, despite great effort to include physicians and advance practice providers from various specialties, findings may be limited by the exclusion of other specialties and health professionals such as nurses and social workers. ACP group visits had never 
been done at this institution before the larger study; however, we did not specifically ask participants if they had any prior experience with ACP group visits that could influenced their responses. Demographic information such as racial and ethnic identity, gender identity, and years in practice were not collected and would have helped to describe our sample and possibly provide some context for their responses. Finally, despite our interview protocol focusing participants' attention and responses to heart failure, it was unclear at times if some participants' responses were related to heart failure or advanced illness, in general. This may limit applicability of our findings to heart failure, specifically; however, findings may still hold important implications for other life-limiting, noncancer conditions characterized by a variable disease trajectory.

\section{Practice Implications}

Despite several limitations, this study sheds light on an important topic and, corroborated by prior research, suggests key considerations for conducting group visits for ACP among older adult patients with heart failure and their caregivers. Clinicians feel positively about group visits and view them as opportunities to have conversations they may not be having during clinic visits as well as provide education about ACP within the context of a disease trajectory. Certain patients may be better candidates for these group visits as perceived by clinicians and could thus be targeted in outreach efforts. Clinician referrals are key to sustaining ACP group visit programs, and results of this study suggest that clinicians support model of care and education for patients. Connecting the dots between referring clinicians and $\mathrm{ACP}$ group visits could result in measurable outcomes related to ACP such as scheduling subsequent ACP discussions with PCPs, naming proxies, and completing advance directive documents.

The authors would like to thank each of the clinicians that generously gave their time and input on this study. Research reported in this publication was supported by the National Institute on Aging of the National Institutes of Health under award R21-AG053716. The content is solely the responsibility of the authors and does not necessarily represent the official views of the National Institutes of Health.

To see this article online, please go to: http://jabfm.org/content/ 34/2/375.full.

\section{References}

1. Lum H, Sudore RL. Advance care planning and goals of care communication in older adults with cardiovascular disease and multi-morbidity. Clin Geriatr Med 2016;32:247-60.

2. Sudore RL, Fried TR. Redefining the "planning" in advance care planning: preparing for end-of-life decision making. Ann Intern Med 2010;153:256-61.

3. Tung EE, North F. Advance care planning in the primary care setting: a comparision of attending staff and resident barriers. Am J Hosp Palliat Care 2009;26:456-63.

4. Ahluwalia SC, Bekelman DB, Huynh AK, Prendergast TJ, Shreve S, Lorenz KA. Barriers and strategies to an integrative model of advance care planning communication. Am J Hosp Palliat Care 2015;32:817-23.

5. Selman L, Harding R, Beynon T, et al. Improving end-of-life care for patients with chronic heart failure: "let's hope it'll get better, when I know in my heart of hearts it won't." Heart 2007;93:963-7.

6. Harding R, Selman L, Beynon T, et al. Meeting the communication and information needs of chronic heart failure patients. J Pain Symptom Manage 2008;36:149-56.

7. Fried TR, O'Leary J, Van Ness P, Fraenkel L. Inconsistency over time in the preferences of older persons with advanced illness for life-sustaining treatment. J Am Geriatr Soc 2007;55:1007-14.

8. Beck A, Scott J, Williams P, et al. A randomized trial of group outpatient visits for chronically ill older HMO members: the cooperative health care clinic. J Am Geriatr Soc 1997;45:543-9.

9. Scott JC, Conner DA, Venohr I, et al. Effectiveness of a group visit model for chronically ill older health maintenance organization members: a 2-year randomized trial of the cooperative health care clinic. J Am Geriatr Soc 2004;52:1463-70.

10. Lum HD, Sudore RL, Matlock DD, et al. A group visit initiative improves advance care planning documentation among older adults in primary care. $\mathrm{J}$ Am Board Fam Med 2017;30:480-90.

11. Lum HD, Jones J, Matlock DD, et al. Advance care planning meets group medical visits: the feasibility of promoting conversations. Ann Fam Med 2016;14:125-32.

12. Bandini JI, Coulourides Kogan A, Olsen B, et al. Feasibility of Group Visits for Advance Care Planning Among Patients with Heart Failure and Their Caregivers. J Am Board Fam Med 2021;34(1):171-180.

13. Tong A, Sainsbury P, Craig J. Consolidated Criteria for Reporting Qualitative Research (COREQ): a 30item checklist for interviews and focus groups. Int $\mathrm{J}$ Qual Health Care 2007;19:349-57.

14. Strauss A. Qualitative analysis for social scientists. Cambridge, England: Cambridge University Press; 1987. 
15. Strauss A, Corbin J. Basics of qualitative research: grounded theory, procedures and techniques. Newbury Park, CA: Sage; 1998.

16. Miles MB, Huberman AM, Saldaña J. Qualitative data analysis: a methods sourcebook. 3rd ed. Thousand Oaks, CA: Sage Publishers; 2013.

17. Zapata C, Lum HD, Wistar E, Horton C, Sudore RL. Feasibility of a video-based advance care planning website to facilitate group visits among diverse adults from a safety-net health system. J Palliat Med 2018;21:853-6.

18. Rabow MW, McGowan M, Small R, Keyssar R, Rugo HS. Advance care planning in community: an evaluation of a pilot 2-session, nurse-led workshop. Am J Hosp Palliat Care 2019;36:143-6.

19. Myers JM, Duthie E, Denson K, Denson S, Simpson D. What can a primary care physician discuss with older patients to improve advance directive completion rates? A clin-IQ. J Patient Cent Res Rev 2017;4:42-5.

20. Ambardekar AV, Thibodeau JT, DeVore AD, et al. Discordant perceptions of prognosis and treatment options between physicians and patients with advanced heart failure. JACC Heart Fail 2017;5:663-71.
21. Hupcey JE, Kitko L, Alonso W. Patients' perceptions of illness severity in advanced heart failure. J Hosp Palliat Nurs 2016;18:110-4.

22. Chandar M, Brockstein B, Zunamo A, et al. Perspectives of health-care providers towards advance care planning in patients with advanced cancer and congestive heart failure. Am J Hosp Palliat Care 2017;34:423-9.

23. Institute of Medicine. Dying in America: Improving quality and honoring individual preferences near the end of life. 2014.

24. Ramsaroop SD, Reid MC, Adelman RD. Completing an advance directive in the primary care setting: what do we need for success? J Am Geriatr Soc 2007;55:277-83.

25. Dillon E, Chuang J, Gupta A, et al. Provider perspectives on advance care planning documentation in the electronic health record: the experience of primary care providers and specialists using advance healthcare directives and physician orders for life-sustaining treatment. Am J Hosp Palliat Care 2017;34:918-24.

26. Johnson M, Janssen D. Advance care planning: a "hard" skill for cardiology clinicians? Heart 2019;105: 1298-9. 


\section{Appendix 1. Research Protocol}

1. Thinking about your older adult patients who have advanced illness (or: advanced heart failure), how often would you say you have advance care planning discussions with them?

a. What types of ACP topics do you discuss with them? (PROBES: future state, prognosis/trajectory, advance directives, surrogate decision-maker, treatment preferences, values/goals for care).

2. What usually prompts you to broach these topics with your patients? (PROBES: change in health status, new diagnosis or prognosis, family request, family conflicts, decision-making capacity)

3. What are some of the challenges you experience when discussing ACP with your older adult patients with advanced illness? (PROBES: initiating the conversation, specific topics that are hard to raise, lack of time, patient barriers, knowledge, personal comfort)

As we mentioned, we have been testing whether group visits-where patients with similar conditions/ disease trajectories (in this case, heart failure) and their caregivers can come together to explore and discuss their values and goals for health care, their general treatment preferences, the need for a surrogate decision maker - are feasible and useful.

In part, we want to know whether attending such a group visit might help patients be more prepared for a tailored one-on-one discussion with their primary provider regarding advance care planning in the context of their specific illness.
4. We are interested in your thoughts on this. In what ways do you think such a group visit could be helpful? (PROBES: strengths and weaknesses of a group visit approach to $\mathrm{ACP}$ )

5. What kinds of topics do you think would be most helpful and appropriate for us to cover in such a group visit?

6. What topics do you think would not be appropriate for a group setting, and why? (PROBBES: What would be more appropriate for a doctor-patient conversation and why?)

7. What would make these group visits helpful to you, in your care of older adult patients or patients with advanced illness? For example, what should we talk about in these visits, to offset your time and effort during a clinic visit?

8. What do you think would be the optimal link between these group visits and your care of the patient? What information regarding the visit would you want to have? (PROBES: direct communication between GV facilitator and provider; summary note)

9. What kinds of patients do you think would benefit most from a group visit on ACP? (PROBES: Can you think of patients you would refer to this group visit?)

10. What kinds of patients would not be appropriate for such a group visit? (PROBES: Think of the kinds of patients you would not refer to this group visit.) 
Appendix 2. Application of Grounded Theory to Research Design and Analysis. Abbreviations: ACP, Advance care planning; GV, group visit.

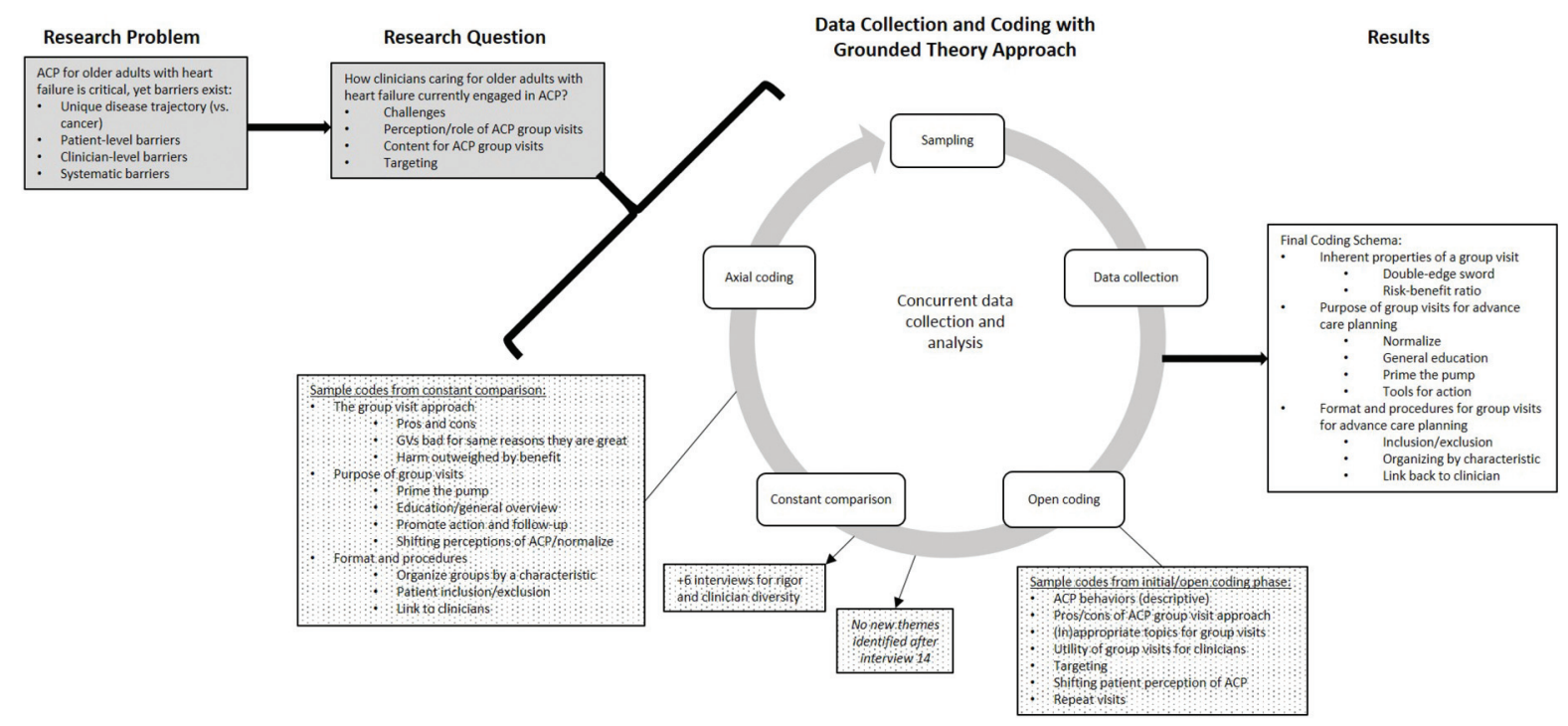

